\title{
St. Gallen/Vienna 2019: A Brief Summary of the Consensus Discussion on the Optimal Primary Breast Cancer Treatment
}

\author{
Marija Balic ${ }^{a}$ Christoph Thomssen ${ }^{b}$ Rachel Würstlein ${ }^{c}$ Michael Gnant ${ }^{d}$ \\ Nadia Harbeck ${ }^{c}$ \\ aDivision of Oncology, Department of Internal Medicine, Medical University Graz, Graz, Austria; ${ }^{\mathrm{b}}$ Department of \\ Gynecology, Martin Luther University of Halle-Wittenberg, Halle (Saale), Germany; ${ }^{{ }^{B} B r e a s t}$ Center, Department \\ of Obstetrics and Gynecology, University of Munich (LMU), Munich, Germany; ${ }^{\mathrm{d} D e p a r t m e n t}$ of Surgery, Medical \\ University of Vienna, Vienna, Austria
}

\section{Keywords}

Early breast cancer - Neoadjuvant systemic therapy ·

Adjuvant treatment - Endocrine therapy · Chemotherapy · Bisphosphonates - Denosumab - Breast surgery · Sentinel node $\cdot$ Axillary dissection - Radiotherapy - Targeted therapy

\section{Abstract}

This year, the St. Gallen Consensus Conference on early breast cancer treatment standards took place for the third time in Vienna, Austria, which is where the next conference will also take place (next date: March 17-20, 2021!). Once again, more than 3,000 participants from over 100 countries came together, and, overall, the 2019 St. Gallen/Vienna conference was a great success. After 3 days of reviews conducted by a global faculty concerning the most important evidence published in the last 2 years, the Consensus votes' challenge was to define the impact on routine everyday practice. This year, the conference's main theme was the optimization of early breast cancer therapies by assessment of the magnitude of benefit, aiming at further refinement when compared to de-escalation and escalation, which were mainly the topic of the 2017 conference. Patient empowerment and the importance of shared decision-making were particularly emphasized. The traditional panel votes were moderated by Eric Winer from Harvard, and for the most part, they managed to clarify most of the critical questions. This brief report by Editors of Breast Care summarizes the results of the 2019 international panel votes with respect to locoregional and systemic treatment as a quick news update for our readers, but it expressly does not intend to replace the official St. Gallen Consensus publication that will follow shortly in Annals of Oncology.

(c) 2019 S. Karger AG, Basel

\section{Introduction}

The St. Gallen Consensus Conference 2019 (March 20-23) was held in Vienna, Austria, for the third time. Giuseppe Curigliano and Eric Winer chaired the panel discussion and the voting. Once again, the conference focused on therapy recommendations and treatment optimization for early breast cancer based on evidence as well as the clinical expertise of the international faculty from 23 countries from all 5 continents (Table 1). As usual, the panel openly disclosed any potential conflicts of interest (COI; www.oncoconferences.ch); the COI committee was again chaired by Harold Burstein (Boston, MA, USA). It was recognized that individual panel members may have financial relationships with commercial entities engaged in research, innovation, and education. None of the declared conflicts were judged as substantially impacting the voting procedure or warrant exclusion of a panel member. However, members with a specific COI were asked to abstain from voting on certain questions.

About 3,000 participants from 105 countries saw 3 days of high-level educational lectures addressing local

\section{KARGER}

(C) 2019 S. Karger AG, Basel 
Table 1. Participants in the St. Gallen/Vienna 2019 Consensus Panel

Conference Co-Chairs

Harold J. Burstein (USA)

Marco Colleoni (Italy)

Giuseppe Curigliano (Italy)

Carsten Denkert (Germany)

Peter Dubsky (Switzerland)

Sibylle Loibl (Germany)

Martine Piccart-Gebhart (Belgium)

Philip Poortmans (The Netherlands/France)

Meredith Regan (USA)

Hans-Jörg Senn (Switzerland)

Beat Thürlimann (Switzerland)

\section{Consensus Moderator}

Eric P. Winer, USA, Dana Farber Cancer Institute, Boston, MA, USA

\section{Panelists}

Fabrice André (France)

Jonas Bergh (Sweden)

Hervé Bonnefoi (France)

Sara Y. Brucker (Germany)

Fatima Cardoso (Portugal)

Lisa Carey (USA)

Eva Ciruelos (Spain)

Marco Colleoni (Italy)

Suzette Delaloge (France)

Carsten Denkert (Germany)

Angelo Di Leo (Italy)

Bent Ejlertsen (Demark)

Florian Fitzal (Austria)

Prudence Francis (Australia)

Viviana Galimberti (Italy)

Heba Gamal (Egypt)

Judy Garber (USA)

William J. Gradishar (USA)

Karen Gelmon (Canada)

Bahadir Gulluoglu (Turkey)

Nadia Harbeck (Germany)

Daniel F. Hayes (USA)

Chiun-Sheng Huang (Taiwan)

Clifford Hudis (USA)

Jens Huober (Germany)

Zefel Jiang (PR China)

Per Karlsson (Sweden)

Eun Sook Lee (South Korea)

Sybille Loibl (Germany)

Monica Morrow (USA)

C. Kent Osborne (USA)

Olivia Pagani (Switzerland)

Ann Partridge (USA)

Martine Piccart-Gebhart (Belgium)

Philipp Poortmans (The Netherlands)

Kathleen Pritchard (Canada)

Meredith Regan (USA)

Emiel J.T. Rutgers (The Netherlands)

Felix Sedlmayer (Austria)

Vladimir Semiglazov (Russia)

Zhiming Shao (PR China)

Ian Smith (UK)

Petra Tesarova (Czech Republic)

Beat Thürlimann (Switzerland)

Masakazu Toi (Japan)

Andrew Tutt (UK)

Giuseppe Viale (Italy)

Toru Watanabe (Japan)

Thimothy Whelan (Australia)

Binghe Xu (PR China) and systemic therapy as well as imaging issues. Furthermore, a great proportion of the sessions dealt with biology, pathology, and translational research issues, providing a basis for future clinical trials and defining the areas of greatest need. Again, a large number of participants from overseas, particularly China, Japan, and the USA, attended the meeting and were actively involved. As a result, the meeting remains the most important international breast cancer meeting on European soil from a global perspective.

The Saturday morning consensus panel was again cochaired by Giuseppe Curigliano (Italy) and Eric Winer (USA), who again moderated the discussion and the voting. 240 questions had been developed and exchanged by panelists upfront in an attempt to reduce debate and resolve controversies regarding clinically important questions.

Clinical trials were agreed to provide the evidence needed for general recommendations on clinical decision-making; however, it was also stated that evidence from randomized clinical trials does not cover all controversies that arise when treating individuals. Thus, expert opinion had to be used when data were lacking. This is the unique feature of the St. Gallen International Consensus.

The panelists were asked to either cast their vote using three possible answers (yes/no/abstain) or, due to the complexity of some questions, choose between more options as possible answers (up to 5). "Abstain" was to be used in cases of insufficient data, a lack of personal expertise on the issue in question, or a given panelist's COI. After each vote, the answers were summarized in percentages. In this report, we summarize the original voting questions and the resulting percentages of the St. Gallen/ Vienna panel discussion on Saturday, March 23, 2019. Abstaining votes and/or very low percentages are not always described in this report.

\section{Surgery of the Primary Tumor, Including after Primary Systemic Therapy}

In general, the panel was inclined to allow further deescalation of surgery in specific situations: the basic requirements were confirmed from previous Consensuses, with the panel declining the notion that requirements for breast-conserving surgery (with radiotherapy planned) should be stricter for lobular cancers (yes 22\%, no 73\%). The voting was less clear on the question of patients with an extensive intraductal component (yes $31 \%$, no $62 \%$ ). There was no real consensus on the management of "focally involved margins," and several highly regarded surgeons warned that forgoing the principles of radicality could send a dangerous message. 
After "no tumor on ink" had finally been firmly established in 2017 as the standard for unifocal residual breast cancers and breast-conserving procedures, this year, a majority of the panel voted that such an approach may also be used for multifocal residual disease (provided that breast radiotherapy is planned) (yes 83\%).

In addition, skin-sparing and nipple-sparing techniques are becoming increasingly popular, and they were the topic of this year's discussions: the panel declined these kinds of options for patients with baseline inflammatory breast cancer, even when a complete clinical response is achieved (83\%); the panel was also split on whether imaging-derived tumor proximity to the skin should exclude such procedures (yes $38 \%$, no $40 \%$ ), as well as centrally located tumors near the nipple (yes $38 \%$, no $43 \%)$.

\section{Management of the Axillae}

By a slim margin, a majority of the panel voted to support forgoing even sentinel lymph node biopsy (SLNB) in specific low-risk or comorbidity situations (such as T1 luminal A, age $>70$ years, comorbidities: yes $56 \%$, no $40 \%$ ) but insisted that in such exceptional cases, axillary ultrasound should be mandatory in complementing clinical palpation $(80 \%)$. The panel declined such SLNB omission for any T2 tumor (89\%).

When asked about the application of the "Z011" criteria in clinical practice, $29 \%$ felt that axillary radiotherapy should be added in all cases, $25 \%$ stated that axillary radiotherapy should be added in cases of aggressive histology such as triple-negative breast cancer (TNBC), and $42 \%$ said that axillary radiotherapy is not necessary at all. For the first time, the panel extended the strategies for 1-2 positive sentinel nodes (SNs), which were derived from trials such as Z011 for breast conservation, to be applicable to mastectomy situations (e.g., 1-2 positive nodes, TNBC, regional nodal irradiation [RNI] planned: yes $71 \%$, no $23 \%$; $1-2$ positive nodes, ER+ or HER $2+$, RNI planned: yes $83 \%$ ), but there was no clear vote on the details: while $48 \%$ of the panelists felt that axillary radiotherapy should be given in accordance with the AMAROS approach in such situations, $8 \%$ felt that this depends on tumor biology (e.g., ER+ vs. TNBC), and 17\% insisted on following with axillary lymph node dissection (ALND). In the absence of RNI, ALND must still be done for mastectomy and $1-2$ positive SNs (66\%).

For patients who are clinically node positive at baseline ( $\mathrm{cN} 1$ ) but downstaging via primary systemic therapy (PST), the panel was split on whether ALND should be done in situations where only 1 or 2 SNs were obtained (yes 44\%, no 54\%), but it endorsed the omission of ALND when $\geq 3$ negative SNs were retrieved (yes 92\%). How- ever, the panel also recommends ALND in $\mathrm{cN} 1 \rightarrow$ PST $\rightarrow$ cN0 situations when a micrometastasis has been found in $1 \mathrm{SN}$ (yes 64\%, no $25 \%$ ).

\section{Radiotherapy}

The panel indicated that hypofractionated breast irradiation can be used for most patients as a care standard (52\% for all patients, $19 \%$ following breast conservation only, and $21 \%$ abstention). With respect to radiotherapy on the breast and on regional lymph nodes (LNs), hypofractionated radiotherapy did not find a clear consensus (36\% for most patients, age $>50$ years $30 \%$, and $30 \%$ abstention).

The panel endorsed (accelerated) partial breast irradiation for patients with low-risk features according to ASTRO/GEC-ESTRO guidelines by a small majority (44\%), with some indicating that this may also be done for intermediate/cautionary risk features (18\%), but $21 \%$ felt that (accelerated) partial breast irradiation should not be the standard because of worse cosmetic outcomes and/ or higher recurrence risks.

The panel clearly agreed that irradiation should be applied to regional nodes in all patients with $\geq 4$ positive nodes (94\%), but in cases of $1-3$ positive nodes, a majority (56\%) indicated that RNI should only be administered if the present features are poor (e.g., TNBC, residual disease after PST), while $29 \%$ felt that RNI should be indicated for all patients with 1-3 positive nodes.

RNI was also decided on as a standard by $44 \%$ of the panelists for patients with $\mathrm{cN} 1 \rightarrow$ PST situations when post-PST SLNB has retrieved a negative SN, while 23\% felt that RNI should only be indicated if risk factors are present, and $17 \%$ did not think RNI should be a standard in such situations.

With regard to post-mastectomy radiotherapy (PMRT; chest wall and RNI), the panel was divided concerning pT3 pN0 situations (yes 56\%, no $44 \%$ ) and N+ 1-3 ER+ or HER2 + (yes 43\%, no 43\%), but clear on N+ 1-3 with adverse features such as TNBC (yes $85 \%$, no $8 \%$ ) and pT2 pN0 with bad features (yes $28 \%$, no $64 \%$ ). For patients with 1-2 positive nodes but no axillary dissection, the panel recommended PMRT + RNI (yes 66\%, no $17 \%)$.

For patients who have had mastectomy and immediate breast reconstruction, the panel stated that PMRT indications should remain the same as for those without immediate breast reconstruction (75\%). For elderly patients with stage $1 \mathrm{ER}+$ disease, the majority was not prepared to leave out radiotherapy after breast conservation (58\%), but the percentage rose to $62 \%$ of the panelists willing to forgo radiotherapy in such cases when the age limit was set to 80 years. 


\section{Pathology}

Prognostic and predictive biomarkers were mainly discussed with the respective therapeutic topics, and only very few markers were voted on separately. Regarding tumor-infiltrating lymphocytes (TILs), $66 \%$ of the panelists recommend routine characterization and reporting. Yet, a majority of $90 \%$ would not decide upon indication for or de-escalation $(79 \%)$ of chemotherapy based on high TILs. Asked for routine TIL analysis in their own clinics, only $28 \%$ of the panelists stated that they routinely have TILs reported.

In contrast to advanced breast cancer, the majority of the panel did not recommend routine reporting of PD-L1 ( $80 \%$ no) or PD-L1ic (92\% no) in early breast cancer.

Regarding multigene signatures and chemotherapy decision-making, $93 \%$ of the experts use genomic assays for determining whether or not to recommend chemotherapy in ER+ HER2 - N0 tumors. In T3 N0 cases, the percentage went down to $74 \%$. More interestingly, in any $\mathrm{T}$ and $1-3$ positive nodes, $78 \%$ of the experts classify multigene signatures as valuable for chemotherapy decision-making. In luminal A-like tumors (ER+ HER2G1) and low-risk multigene signatures, $65 \%$ of the panel voted for a recommendation for chemotherapy only in the infrequent case of $\geq 4$ involved LNs. TAILORx and MINDACT implications are discussed below in the context of decision-making for chemotherapy indication.

Regarding the HR status, it was stated that there is a need for better evaluation of ideal cut-offs for prescription of endocrine therapy for ER+ tumors, mainly with ER levels <10\%: $24 \%$ of the experts did not see an ideal cut-off for ER status, whereas $38 \%$ would recommend prescription with levels of $\geq 10 \%$.

\section{Adjuvant Endocrine Therapy}

\section{Adjuvant Endocrine Therapy for \\ Premenopausal Patients}

Most experts (68.1\%) opted for prior chemotherapy as a clinicopathological indication to provide ovarian function suppression (OFS) to premenopausal patients; $84.8 \%$ felt that a young age $\leq 35$ years alone was sufficient as an indication. A moderate risk itself without chemotherapy administration was not felt to be a strong enough indication ( $45.8 \%$ yes, $41.7 \%$ no). $37.8 \%$ felt that involvement of $\geq 1 \mathrm{LN}$ was a strong enough indication for OFS, and only $17.8 \%$ voted for $\geq 4$ LNs. For $59.6 \%$, an adverse result in a multigene assay would be an indication for OFS, whereas a HER2+ status would not be sufficient for $52.1 \%$. In a 33 -year-old patient ( $\mathrm{pN}+\mathrm{ER}+\mathrm{PR}+\mathrm{G} 3$, adjuvant chemotherapy planned), $57.1 \%$ would opt for OFS plus either tamoxifen or an aromatase inhibitor (AI) depending on tolerance. $55.1 \%$ considered 5 years as the appropriate duration for OFS $[1,2]$.

\section{Adjuvant Endocrine Therapy for}

\section{Postmenopausal Patients}

The vast majority of experts (95.7\%) would consider an AI at some point of treatment for their postmenopausal patients. Grade $\geq 3 \mathrm{Ki}-67$ would be a parameter for inclusion of an AI for $83 \%$ and HER $2+$ for $68.1 \%$. The majority $(59.2 \%)$ stated that they would not start upfront with an AI for all patients, but $93.8 \%$ would do this for patients at higher risk by stage.

\section{Duration of Adjuvant Endocrine Therapy}

In premenopausal women with stage 1 disease, $54.3 \%$ would stop tamoxifen after 5 years, whereas $79.6 \%$ would continue up to 10 years with stage $2(\mathrm{~N}+)$ or stage 3 disease.

In postmenopausal patients with stage 1 disease, $72.3 \%$ would not continue endocrine therapy after 5 years of tamoxifen, and $78.3 \%$ would not do so after 5 years of an AI. With stage 2 (N0) disease, $68.1 \%$ would continue endocrine therapy after 5 years of tamoxifen, but $59.2 \%$ would not after 5 years of an AI. With stage $2(\mathrm{~N}+)$ disease, $97.9 \%$ would continue endocrine therapy after 5 years of tamoxifen, and $81.2 \%$ after 5 years of an AI. Overall, 58\% stated that extended adjuvant therapy should last for 10 years, and $31.7 \%$ opted for $7-8$ years. With veryhigh-risk disease (e.g., $\geq 10$ involved LNs), $60.4 \%$ would decide on whether to continue endocrine therapy beyond 10 years on a case-by-case basis.

\section{Neoadjuvant Endocrine Therapy}

If neoadjuvant therapy was considered for a postmenopausal patient with a luminal A-like tumor, $81.2 \%$ would opt for neoadjuvant endocrine therapy. $46.9 \%$ stated that the optimal duration for neoadjuvant endocrine therapy was the time it took for optimal reduction in tumor size, and $32.7 \%$ thought at least 6 months.

\section{Adjuvant and Neoadjuvant Chemotherapy}

Whether or not chemotherapy should be indicated for luminal A (ER+, HER2-, G1) breast cancer depends on the positivity of locoregional LNs. According to $20.8 \%$ of the panelists, chemotherapy should be provided to patients with $2-3$ positive LNs, as well as to patients with 4-9 positive LNs according to the majority (64.6\%). The results were very similar to the decision made regarding lobular cancer. According to the TAILORx study, the panel voted on indication for chemotherapy for women younger than 50 years with node- 
negative cancer and a Recurrence Score (RS) of 21-25. $41.7 \%$ of the panelists voted for chemotherapy and endocrine therapy, $25 \%$ for OFS and endocrine therapy, $10.4 \%$ for the combination of chemotherapy, OFS, and endocrine therapy, and $16.6 \%$ for tamoxifen only. Regarding postmenopausal women with an RS $>26,57.1 \%$ voted for chemotherapy for selected patients, depending on other histopathologic characteristics and patients' preferences. $38.8 \%$ of the panelists felt that chemotherapy should be routinely administered to this population, and only $4.1 \%$ supported chemotherapy only for an RS $>30$ [3]. 78.7\% agreed that for patients with an RS $<11$ aged $\geq 50$ years and having $1-2$ positive LNs, chemotherapy may be omitted, according to the PlanB Trial [4]. Similarly, in MammaPrint-low patients aged $>50$ years with 1-2 positive LNs, based on the MINDACT data, $80.9 \%$ of the panelists recommend forgoing chemotherapy. Age does not play a critical role, as even for the group of patients $<50$ years with low MammaPrint results, $78.7 \%$ of the panelists voted against an indication for chemotherapy [5].

For the luminal A subtype based on IHC, when neoadjuvant treatment is considered for postmenopausal patients, $81.2 \%$ of the panelists voted for endocrine neoadjuvant treatment instead of chemotherapy, and according to the majority of the panel, this treatment should be administered over periods of $\geq 6$ months, until optimal reduction of the tumor is achieved, regardless of how long it takes (32.7\% voted for 6 months, and $46.9 \%$ for even longer, as long as it needs to achieve an optimal response). For ER+ breast cancers, a majority of the panelists (55.4\%) choose alkylators and taxanes (i.e., the TC regimen [4]) over combinations of anthracyclines, alkylators, and taxanes $(31.2 \%)$. This overall vote will probably be discussed in further detail in the official St. Gallen Consensus publication, where we expect that this will be addressed according to risk stratification and shared decision-making.

In TNBC, the roles of both anthracyclines and taxanes remain clear. $77.6 \%$ of the panelists determined anthracyclines, alkylators, and taxanes as the preferred regimen, and only $16.3 \%$ supported the use of alkylators and taxanes only. However, for stage 1 TNBC, $52.2 \%$ chose to use only alkylators and taxanes, while $30.4 \%$ preferred the combination of anthracyclines, alkylators, and taxanes (and this result represented a shift towards an anthracycline-free regimen when compared to the 2017 St. Gallen Consensus Meeting [6], when only 55.8\% agreed [no 40.4\%] with regimens containing anthracyclines as well as taxanes for stage 1 disease). For stages 2 and 3 , the percentage of the panelists opting for anthracyclines, alkylators, and taxanes was $93.3 \%$.

53.1\% did not accept the use of a platinum-based regimen in the neoadjuvant treatment of all TNBC patients.
The panelists discussed this result, and there was some disagreement on the use of platinum depending on stage and disease risk, due to the consistency of improvement of pathologic complete response ( $\mathrm{pCR}$ ) throughout the studies. However, this could not be clarified through additional voting, and it will hopefully be discussed in more detail in the official St. Gallen Consensus publication. The vote on the use of platinum in the neoadjuvant setting in $B R C A$-mutated patients turned out to be somewhat disappointing, as $67.3 \%$ of the panelists voted to include platinum in neoadjuvant treatment despite recent evidence suggesting that, in the neoadjuvant setting, there was no increase in pCR depending on the BRCA status. While this was discussed, it was clarified no further, and we can only wait for clarification in the official St. Gallen Consensus publication. Even when the vote was repeated, the proportion of panelists supporting the general use of platinum did not change significantly.

Regarding adjuvant treatment of patients diagnosed with very small TNBC $(<6 \mathrm{~mm}$, pT1aN0), a majority of the panelists $(65.3 \%)$ supported the occasional recommendation of adjuvant chemotherapy. If CMF is an option at any stage, for $6.5 \%$ of the panelists it would be IV administration every 3 weeks for $6-8$ cycles; $26.1 \%$ would opt for oral administration, with IV administration on days 1 and 8 of the 4 -week cycle for 4-6 cycles, while $10.9 \%$ would choose days 1 and 8 of the 4 -week cycle for IV administration, also for $4-6$ cycles. $26.1 \%$ accepted any of the CMF options as reasonable.

For T1a HER2+ early breast cancer, $42.6 \%$ of the panelists supported anti-HER2 therapy, while $55.6 \%$ did not. However, $61.7 \%$ agreed that the ER status does not affect any of the thresholds. For stage 1 disease, $73.5 \%$ supported TH adjuvant treatment (the correct abbreviation should be TT, for taxane and trastuzumab), $4.1 \%$ supported THP treatment, and only $12.2 \%$ supported AC treatment followed by $\mathrm{TH}$ (with or without $\mathrm{P}$ ).

In stages $2(\mathrm{~N}+)$ and 3 , the most preferred options were $\mathrm{AC}$ or EC followed by taxane in combination with $\mathrm{T}$ and $\mathrm{P}$, and $14.3 \%$ chose docetaxel and carboplatin with $\mathrm{T}$ and P. Only $4.1 \%$ preferred AC or EC followed by taxane and trastuzumab only. In neoadjuvant treatment, $52.1 \%$ did not support pertuzumab in the combination with trastuzumab for stage 1 disease. Moreover, while $48.9 \%$ of the panelists stated that pertuzumab should not be added for the treatment of stage 1 disease, $25.2 \%$ recommended it for the treatment of stage $1 \mathrm{ER}$ - disease. In stage $2(\mathrm{~N}+)$ and stage $3,76.6 \%$ agreed that pertuzumab should be added in all cases [7]. In stages 2 and 3, 19.1\% would recommend pertuzumab in addition to trastuzumab only in ER- disease. 89.9\% agreement was achieved regarding a 12-month duration for trastuzumab. Regarding the 6-month administration, $29.2 \%$ deemed it acceptable for stage 1 patients, but $64.6 \%$ did not support a prior selec- 
tion of patients for the shorter duration. During the discussion, 6 months were mentioned as an acceptable option for patients with a clinical reason for discontinuation. $20.8 \%$ of the panelists would never use neratinib in adjuvant treatment, $20.8 \%$ would use it in all ER+ $\mathrm{N}+$ HER 2+ patients, and $27.1 \%$ would use neratinib in ER+ HER2+ patients with $\geq 4$ positive LNs [8]. $29.2 \%$ abstained from answering the question on adjuvant neratinib.

The panel nearly unanimously (98\%) agreed on indication for neoadjuvant treatment for stage 2 and 3 TNBC and HER2+ patients, which was in line with the 2017 Consensus [6].

Measuring residual cancer after neoadjuvant treatment may be specifically highlighted as a potential assessment for patients at risk and in need of further intensified treatment. For TNBC patients who have received neoadjuvant sequential anthracycline and alkylator chemotherapy followed by taxanes and who have had residual cancer in axillary LNs or the breast $(\geq 1 \mathrm{~cm}$ residual cancer and/or LN+), $83.3 \%$ would prefer to add capecitabine to adjuvant treatment, $6.2 \%$ would not add any treatment, and $4.2 \%$ would choose CMF as adjuvant treatment. In cases with residual cancers $<1 \mathrm{~cm}$ and no residual tumor in LNs, 51\% would still recommend capecitabine and $38.8 \%$ would not recommend further chemotherapy. If residual cancer has been found in a breast and/or axilla after neoadjuvant treatment with $\mathrm{AC} / \mathrm{EC}$ followed by TH (without $\mathrm{P}$ ), the preferred adjuvant systemic therapy would be TDM1 for $91.7 \%$ of the panelists. When neoadjuvant treatment has encompassed TCHP or AC/EC followed by taxane with $\mathrm{H}$ and $\mathrm{P}$, and there is still residual cancer in tumors $>1 \mathrm{~cm}$ and/ or an axilla, again, $93.9 \%$ of the panelists would recommend TDM1 [9].

In the case of pCR after neoadjuvant therapy for HER2+ disease with a primary positive axilla, $38.6 \%$ of the panelists would recommend trastuzumab, $47.7 \%$ would recommend trastuzumab and pertuzumab, and 9.1\% would recommend trastuzumab and pertuzumab only for ER- patients. For patients with primary nodenegative disease who have received neoadjuvant treatment with trastuzumab and pertuzumab, $52.2 \%$ would recommend adjuvant trastuzumab, $26.1 \%$ would recommend trastuzumab and pertuzumab, and $13.0 \%$ would prefer the combination of trastuzumab and pertuzumab only for ER- disease.

\section{Adjuvant Bone-Targeted Therapies}

The 2017 St. Gallen Consensus Conference defined adjuvant bisphosphonates intended to improve diseasefree survival (DFS) of postmenopausal patients as a stan- dard of care regardless of bone mineral density according to Dhesy-Thind et al. [10] and the EBCTCG report on adjuvant bisphosphonate treatment [11]. This year, the vote on the use of bisphosphonates in premenopausal patients on ovarian suppression with either tamoxifen or AIs was very similar to the 2017 vote $(53.1 \%$ yes vs. $36.7 \%$ no in $2019 ; 53 \%$ yes and $37 \%$ no in 2017). For postmenopausal patients, the panel strongly supported the use of bisphosphonates to improve DFS, with a "yes" from $83.7 \%$ and a "no" from only $8.2 \%$. However, when asked how frequently in the clinical routine they use bisphosphonates, only $42.6 \%$ responded "yes," confirming that they were using it, and $40.4 \%$ responded "no." The panelists were clear in stating that denosumab $60 \mathrm{mg}$ twice a year should not be used as a substitute for bisphosphonate ( $12.5 \%$ yes, $75 \%$ no) as suggested by the ABCSG-18 Trial [12], despite a recent publication on the positive impact on DFS [13].

\section{Fertility Preservation and Pregnancy after Breast Cancer}

91.5\% of the panelists supported OFS for patients with ER- disease who wanted to be pregnant in the future, and only $2.5 \%$ did not. For patients with ER+ disease, the proportion of the panelists who supported OFS was slightly smaller, but it was still a majority, with $79.6 \%$ in favor of OFS and $12.2 \%$ not supporting it.

For patients who are planning on becoming pregnant within 5 years following surgery, the panel did not support interruption of endocrine therapy at any time $(79.2 \%$ no vs. $16.7 \%$ yes), but after 18 months of endocrine therapy, $78.0 \%$ believed that interruption of endocrine therapy is reasonable, whereas $17.1 \%$ did not. The majority of the panel was in favor of the scheme of the ongoing POSITIVE Trial, and hopefully most of the interrupted endocrine treatments and successful pregnancies will occur within this trial and evidence will be provided in the future.

\section{Genetic Testing}

In the discussion on providing genetic counselling, the panelists agreed that the proportion of breast cancer patients who should be tested is increasing, while there is still potential for improvement. However, the majority of the panelists did not support the idea of all women with breast cancer being offered genetic counselling; $70.8 \%$ did not agree that this is a good approach, whereas $29.2 \%$ of the panelist were in favor of testing all women with breast cancer. With a more individualized approach, the proportion of the panelists in favor of ge- 
netic testing increased. For breast cancer patients with a clear family history with the disease, $100 \%$ of panelists voted in favor of genetic counselling. For patients aged $<35$ years at diagnosis, $95.9 \%$ of the panel would have advised genetic counselling, while only $4.1 \%$ would not have done so. For otherwise unselected patients at ages $<50$ years, $65.3 \%$ of the panelists did not agree that genetic counselling should be considered, while $32.7 \%$ did. Concerning patients with TNBC below the age of 60 years, $85.4 \%$ were in favor of genetic counselling and only $14.6 \%$ were not. When asked whether genetic counselling should be considered for TNBC patients at any age, only $38.8 \%$ were in favor of counselling and $59.2 \%$ were not.

\section{Ductal Carcinoma in situ}

It remains a matter of debate if ductal carcinoma in situ (DCIS) should always be subject to surgery, or if there is a risk stratification possibility, seeing that $<50 \%$ of DCIS develop into an invasive cancer later on. During the 16th St. Gallen International Breast Cancer Conference, some agreement on de-escalation could be achieved. The great majority of the panel members (97.9\%) agreed that small DCIS is associated with a better prognosis when detected by screening in patients $<50$ years of age, and that it is associated with favorable pathological features such as grade 1 or 2 or other low-risk features. $84.1 \%$ agreed that when DCIS with favorable prognostic features is removed by surgery and a clear margin of $>5 \mathrm{~mm}$ is achieved, it is reasonable to omit radiotherapy. Only $15.6 \% \mathrm{did}$ not agree to omitting radiotherapy in these cases. $55.6 \%$ of the panelists thought it reasonable to omit endocrine therapy for all patients, whereas $42.2 \%$ found it reasonable for patients with favorable prognostic features. Moreover, for patients with favorable prognostic features and clear margins of $\geq 5 \mathrm{~mm}, 66.7 \%$ voted for no radiotherapy and endocrine therapy, whereas $33.3 \%$ did not find omitting both therapies reasonable.

\section{Lifestyle}

This year, only two questions addressed lifestyle, concerning diet and exercise. Regarding an exercise regimen to be recommended as a standard of care, a great majority of $84.1 \%$ supported this idea, and only $11.4 \%$ of the votes were not in favor. Furthermore, a clear majority of $83.0 \%$ of the panelists were in favor of maintaining a stable weight and recommended that weight gain should be avoided. $14.9 \%$ of the panel members would not give such a recommendation to breast cancer patients.

\section{Conclusions}

The panelists acknowledged that most patients will not benefit from adjuvant therapies in terms of overall survival, defined as the ultimate goal of adjuvant therapies, and many adjuvant treatments only have a small-to-marginal impact. $91.4 \%$ of the panel members agreed that patients should be informed about the rather low magnitude of any benefit from interventions, as well as the fact that offering no treatment is a reasonable alternative on certain occasions. Only $8.5 \%$ of the panelists disagreed. This Consensus was in line with the broadly discussed topic of shared decision-making and patient empowerment.

\section{Disclosure Statement}

Marija Balic reports consulting fees, lecture honoraria, advisory board memberships, and travel grants from Amgen, AstraZeneca, Bayer, Boehringer Ingelheim, Celgene, Lilly, MSD, Novartis, Pfizer, and Roche, as well as research funding from Eli Lilly and Pfizer. Christoph Thomssen reports receiving honoraria for lectures for advisory boards and lectures: Amgen, AstraZeneca, Celgene, Daiichi Sankyo, Eisai, Lilly, MSD, NanoString, Novartis, Pfizer, Puma, and Roche, as well as research support from American Diagnostica, Affymetrix, and NanoString. Rachel Würstlein reports receiving lecture and consulting fees, advisory board memberships, travel grants, and research funding by Agenda, Amgen, AstraZeneca, Boehringer Ingelheim, Carl Zeiss, Celgene, Daiichi Sankyo, Essay, Genomic Health, GSK, Hexal, Eli Lilly, MSD, Mundipharma, NanoString, Novartis, Odonate Therapeutics, Paxman, Palleos, Pfizer, Pierre Fabre, Puma Technology, Riemser, Roche, Sandoz/Hexal, Seattle Genetics, TESARO Bio, and Teva. Michael Gnant reports having received personal honoraria, consulting fees, and travel support from: Amgen, AstraZeneca, Celgene, Eisai, Eli Lilly, Ibsen, Madison, Medtronic, Novartis, NanoString Technologies, Pfizer, and Roche/Genentech. An immediate family member is employed by Sandoz. Nadia Harbeck reports honoraria or consultation fees from the following entities: Agendia, Amgen, AstraZeneca, Celgene, Daiichi Sankyo, Genomic Health, Lilly, MSD, NanoString, Novartis, Odonate Therapeutics, Pfizer, Roche, Sandoz/Hexal, and Seattle Genetics.

\section{References} Ciruelos E, Bellet M, et al.; SOFT Investigators; International Breast Cancer Study Group. Adjuvant ovarian suppression in premenopausal breast cancer. N Engl J Med. 2015 Jan;372(5):436-46.

2 Pagani O, Regan MM, Walley BA, Fleming GF, Colleoni M, Láng I, et al.; TEXT and SOFT Investigators; International Breast Cancer Study Group. Adjuvant exemestane with ovarian suppression in premenopausal breast cancer. N Engl J Med. 2014 Jul;371(2): 107-18.

3 Sparano JA, Gray RJ, Makower DF, Pritchard KI, Albain KS, Hayes DF, et al. Adjuvant Chemotherapy Guided by a 21-Gene Expression Assay in Breast Cancer. N Engl J Med. 2018 Jul;379(2):111-21. 
4 Nitz U, Gluz O, Clemens M, Malter W, Reimer T, Nuding B, et al.; West German Study Group PlanB Investigators. West German Study PlanB Trial: Adjuvant Four Cycles of Epirubicin and Cyclophosphamide Plus Docetael Versus Six Cycles of Docetaxel and Cyclophosphamide in Her2-Negative Eraly Breast Cancer. J Clin Oncol. 2019;37(10): 799-808.

5 Cardoso F, van't Veer LJ, Bogaerts J, Slaets L, Viale G, Delaloge S, et al.; MINDACT Investigators. 70-Gene Signature as an Aid to Treatment Decisions in Early-Stage Breast Cancer. N Engl J Med. 2016 Aug;375(8):717-

6 Gnant M, Harbeck N, Thomssen C. St. Gallen/Vienna 2017: A Brief Summary of the Consensus Discussion about Escalation and De-Escalation of Primary Breast Cancer Treatment. Breast Care (Basel). 2017;12(2): 102-7.
7 von Minckwitz G, Procter M, de Azambuja E, Zardavas D, Benyunes M, Viale G, et al.; APHINITY Steering Committee and Investigators. Adjuvant Pertuzumab and Trastuzumab in Early Her2-Positive Breast Cancer. N Engl J Med. 2017 Jul;377(2):122-31.

8 Martin M, Holmes FA, Ejlertsen B, Delaloge $\mathrm{S}$, Moy B, Iwata H, et al. Neratinib after trastuzumab-based adjuvant therapy in HER2-positive breast cancer (ExteNET): 5 year analysis of a randomised, double-blind, placebo controlled, phase 3 trial. Lancet Oncol. 2017 Dec; 18(12):1688-700.

9 von Minckwitz G, Huang CS, Mano MS, Loibl S, Mamounas EP, Untch M, et al.; KATHERINE Investigators. Trastuzumab Emtansine for Resiudal Invasive HER2-Positive Breast Cancer. N Engl J Med. 2019; 380(7):617-28.

10 Dhesy-Thind S, Fletcher GG, Blanchette PS, Clemons MJ, Dillmon MS, Frank ES, et al. Use of adjuvant bisphosphonates and other bonemodifying agents in breast cancer: a Cancer Care Ontario and American Society of Clinical Oncology clinical practice guideline. J Clin Oncol. 2017 Mar;35(18):2062-81.
11 Early Breast Cancer Trialists' Collaborative Group (EBCTCG). Adjuvant bisphosphonate treatment in early breast cancer: meta-analyses of individual patient data from randomized trials. Lancet. 2015;386(1001):1353-61.

12 Gnant M, Pfeiler G, Dubsky PC, Hubalek M, Greil R, Jakesz R, et al.; Austrian Breast and Colorectal Cancer Study Group. Adjuvant denosumab in breast cancer (ABCSG-18): a multicentre, randomised, double-blind, placebo-controlled trial. Lancet. 2015 Aug;386 (9992):433-43.

13 Gnant M, Pfeiler G, Steger GG, Egle D, Greil $\mathrm{R}$, Fitzal F, et al. Adjuvant denosumab in postmenopausal patients with hormone receptorpositive breast cancer (ABCSG-18): diseasefree survival results from a randomised, double-blind, placebo-controlled, phase 3 trial. Lancet Oncol. 2019;20(3):339-51. 